\title{
New Constructions of Edge Bimagic Graphs from Magic Graphs
}

\author{
Jayapal Baskar Babujee, Babitha Suresh \\ Department of Mathematics, Anna University, Chennai, India \\ E-mail: baskarbabujee@yahoo.com,babi_mit@yahoo.co.in \\ Received September 5, 2011; revised October 14, 2011; accepted October 21, 2011
}

\begin{abstract}
An edge magic total labeling of a graph $G(V, E)$ with $p$ vertices and $q$ edges is a bijection $f$ from the set of vertices and edges to $1,2, \cdots, p+q$ such that for every edge $u v$ in $E, f(u)+f(u v)+f(v)$ is a constant $k$. If there exist two constants $k_{1}$ and $k_{2}$ such that the above sum is either $k_{1}$ or $k_{2}$, it is said to be an edge bimagic total labeling. A total edge magic (edge bimagic) graph is called a super edge magic (super edge bimagic) if $f(V(G))=\{1,2, \cdots, p\}$. In this paper we define super edge edge-magic labeling and exhibit some interesting constructions related to Edge bimagic total labeling.
\end{abstract}

Keywords: Graph, Labeling, Magic Labeling, Bimagic Labeling, Function

\section{Introduction}

A labeling of a graph $G$ is an assignment $f$ of labels to either the vertices or the edges or both subject to certain conditions. Labeled graphs are becoming an increasingly useful family of Mathematical Models from a broad range of applications. Graph labeling was first introduced in the late 1960's. A useful survey on graph labeling by J. A. Gallian (2010) can be found in [1]. All graphs considered here are finite, simple and undirected. We follow the notation and terminology of [2]. In most applications labels are positive (or nonnegative) integers, though in general real numbers could be used. A ( $p$, q)-graph $G=(V, E)$ with $p$ vertices and $q$ edges is called total edge magic if there is a bijection $f: V \cup E \rightarrow$ $\{1,2, \cdots, p+q\}$ such that there exists a constant $k$ for any edge $u v$ in $E, f(u)+f(u v)+f(v)=k$. The original concept of total edge-magic graph is due to Kotzig and Rosa [3]. They called it magic graph. A total edge-magic graph is called a super edge-magic if $f(V(G))=\{1,2, \cdots, p\}$. Wallis [4] called super edge-magic as strongly edgemagic. An Edge antimagic total labeling of a graph with $p$ vertices and $q$ edges is a bijection from the set of edges to $1,2, \cdots, p+q$ such that the sums of the label of the edge and incident vertices are pairwise distinct.

It becomes interesting when we arrive with magic type labeling summing to exactly two distinct constants say $k_{1}$ or $k_{2}$. Edge bimagic totally labeling was introduced by J. Baskar Babujee [5] and studied in [6] as $(1,1)$ edge bimagic labeling. A graph $G(p, q)$ with $p$ vertices and $q$ edges is called total edge bimagic if there exists a bijection $f: V \cup E \rightarrow\{1,2, \cdots, p+q\}$. such that for any edge $u v \in E$, we have two constants $k_{1}$ and $k_{2}$ with $f(u)+f(v)+f(u v)=k_{1}$ or $k_{2}$. A total edge-bimagic graph is called super edge-bimagic if $f(V(G))=\{1,2, \cdots, p\}$. Super edge-bimagic labeling for path, star- $K_{1, n}, K_{1, n, n}$ are proved in [7]. Super edge-bimagic labeling for cycles, Wheel graph, Fan graph, Gear graph, Maximal Planar class- $P I_{n}: \quad n \geq 5$,

$$
\begin{aligned}
& K_{1, m} \cup K_{1, n}(m, n \geq 1), P_{n} \cup P_{n+1}(n \geq 2), P_{m} \odot K_{1, n}, \\
& \left.C_{3} \cup K_{1, n}(n \geq 1), P_{n}+N_{2}(n \geq 3), P_{2} \cup m K_{1}+N_{2}\right)(m \geq 1),
\end{aligned}
$$

$(3, n)$-kite graph $(n \geq 2)$, are proved in [8-10]. In this paper we define super edge edge-magic and exhibit some interesting constructions related to Edge bimagic total labeling. For our convenience, we state total edge-magic as edge-magic total labeling throughout the paper.

\section{Main Results}

On renaming Super edge-magic as Super vertex edgemagic it motivates us to define super edge edge-magic labeling in graphs.

Definition 2.1 A graph $G=(V, E)$ with $p$ vertices and $q$ edges is called total edge magic if there is a bijection function $f: V \cup E \rightarrow\{1,2, \cdots, p+q\}$ such that 
for any edge $u v$ in $E$ we have a constant $k$ with $f(u)+f(u v)+f(v)=k$. A total edge-magic graph is called super edge edge-magic if $f(E(G))=\{1,2, \cdots, q\}$.

Next we introduce a definition for vertex superimposing between two graphs.

Definition 2.2 If $G_{1}\left(p_{1}, q_{1}\right)$ and $G_{2}\left(p_{2}, q_{2}\right)$ are two connected graphs, $G_{1} \hat{o} G_{2}$ is obtained by superimposing any selected vertex of $G_{2}$ on any selected vertex of $G_{1}$. The resultant graph $G=G_{1} \hat{0} G_{2}$ consists of $p_{1}+p_{2}-1$ vertices and $q_{1}+q_{2}$ edges.

Theorems 2.3 If $G$ has super edge edge-magic total labeling then, $G \hat{o} P_{n}$ admits edge bimagic total labeling.

Proof: Let $G(p, q)$ be super edge edge-magic graph with the bijective function $f: V \cup E \rightarrow\{1,2, \cdots, p+q\}$. such that $f(u)+f(u v)+f(v)=k_{1}$. Let $w \in V$ be the vertex whose label $f(w)=p+q$ is the maximum value. Consider the path $P_{n}$ with vertex set

$\left\{x_{i}: 1 \leq i \leq n\right\}$ and edge set $\left\{x_{i} x_{i+1}: 1 \leq i \leq n-1\right\}$. We superimpose one of the pendent vertex of the path $P_{n}$ say $x_{1}$ on the vertex $w \in V$ of $G$. Now we define the new graph called $G^{\prime}=G \hat{o} P_{n}$ with vertex set

$V^{\prime}=V \cup\left\{x_{i}: 2 \leq i \leq n\right\}$ and

$E^{\prime}=E \cup\left\{w x_{2}, x_{i} x_{i+1}: 2 \leq i \leq n-1\right\}$. Consider the bijective function $g: V^{\prime} \cup E^{\prime} \rightarrow\{1,2, \cdots, p+q, p+q+1, \cdots$, $p+q+n, \cdots, p+q+2 n-2\}$ defined by $g(v)=f(v)$ for all $v \in V$ and $g(u v)=f(u v)$ for all $u v \in E$.

From our construction of new graph $G^{\prime}$,

$$
f(w)=g\left(x_{1}\right)=g(w)=p+q .
$$

For $2 \leq i \leq n, g\left(x_{i}\right)= \begin{cases}p+q+\left\lceil\frac{n}{2}\right\rceil+\frac{i-2}{2}, & i \text { even } \\ p+q+\frac{i-1}{2}, & i \text { odd }\end{cases}$

Now

$$
\begin{gathered}
g\left(w x_{2}\right)=p+q+2(n-1) ; \\
g\left(x_{i} x_{i+1}\right)=p+q+2 n-i-1 \text { for } 2 \leq i \leq n-1 .
\end{gathered}
$$

Since the graph $G$ is super edge edge-magic with common count $k_{1}$, implies that $g(u)+g(u v)+g(v)=k_{1}$ for all $u v \in E$. Now we have to prove that the remaining edges in the set

$\left\{w x_{2}, x_{i} x_{i+1}: 2 \leq i \leq n-1\right\}$ have the common count $k_{2}$.

For the edge $w x_{2}$,

$$
\begin{aligned}
& g(w)+g\left(w x_{2}\right)+g\left(x_{2}\right) \\
& =p+q+p+q+2 n-2+p+q+\left\lceil\frac{n}{2}\right\rceil \\
& =3 p+3 q+2 n+\left\lceil\frac{n}{2}\right\rceil-2=k_{2}
\end{aligned}
$$

For any edge $x_{i} x_{i+1}$, if $i$ is even,

$$
\begin{aligned}
& g\left(x_{i}\right)+g\left(x_{i} x_{i+1}\right)+g\left(x_{i+1}\right) \\
& =p+q+\left\lceil\frac{n}{2}\right\rceil+\frac{i-2}{2}+p+q+2 n-i-1+p+q+\frac{i}{2} \\
& =3 p+3 q+2 n+\left\lceil\frac{n}{2}\right\rceil-2=k_{2}
\end{aligned}
$$

If $i$ is odd,

$$
\begin{aligned}
& g\left(x_{i}\right)+g\left(x_{i} x_{i+1}\right)+g\left(x_{i+1}\right) \\
& =p+q+\frac{i-1}{2}+p+q+2 n-i-1+p+q+\left\lceil\frac{n}{2}\right\rceil+\frac{i-1}{2} \\
& =3 p+3 q+2 n+\left\lceil\frac{n}{2}\right\rceil-2=k_{2} \cdots
\end{aligned}
$$

Thus $G^{\prime}=G o ̂ P_{n}$ has two common count $k_{1}$ and $k_{2}$. Hence $G o ̂ P_{n}$ has edge bimagic total labeling.

Example 2.4 Taking $G=K_{1,6}$ which is super edge edge magic, by using the theorem 2.3, $G$ ô $P_{5}=K_{1,6}$ ô $P_{5}$ admits edge bimagic total labeling with two common count $k_{1}=26$ and $k_{2}=50$ is given in Figure 1 .

Theorem 2.5 GôK $K_{1, n}$ is total edge bimagic for any arbitrary super edge edge-magic Graph $G$.

Proof: Let $G(p, q)$ be super edge edge-magic graph with the bijective function $f: V \cup E \rightarrow\{1,2, \cdots, p+q\}$ such that $f(u)+f(u v)+f(v)=k_{1}$. Let $w \in V$ be the vertex whose label $f(w)=p+q$ is the maximum value. Consider the star $K_{1, n}$ with vertex set $\left\{x_{0}, x_{i}: 1 \leq i \leq n\right\}$ and edge set $\left\{x_{0} x_{i}: 1 \leq i \leq n\right\}$. We superimpose the vertex $x_{0}$ of the star $K_{1, n}$ graph on the vertex $w \in V$ of $G$. Now we define the new graph called $G^{\prime}=G \hat{o} K_{1, n}$ with vertex set $V^{\prime}=V \cup\left\{x_{i}: 1 \leq i \leq n\right\}$ and $E^{\prime}=E \cup\left\{w x_{i}: 1 \leq i \leq n\right\}$. Consider the bijective function $g: V^{\prime} \cup E^{\prime} \rightarrow\{1,2, \cdots, p+q, p+q+1, \cdots, p+q+n, \cdots, p+q+2 n\}$ defined by $g(v)=f(v)$ for all $v \in V$ and $g(u v)=f(u v)$ for all $u v \in E$.

From our construction of new graph $G^{\prime}$,

$$
\begin{gathered}
f(w)=g\left(x_{0}\right)=g(w)=p+q . \\
g\left(x_{i}\right)=p+q+2 n-(i-1), \text { for } 1 \leq i \leq n .
\end{gathered}
$$

and

$$
g\left(w x_{i}\right)=p+q+i ; \text { for } 1 \leq i \leq n .
$$

Since the graph $G$ is super edge edge-magic with common count $k_{1}$, implies that $g(u)+g(u v)+g(v)=k_{1}$ for

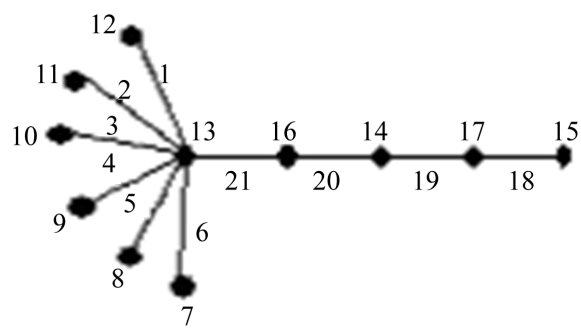

Figure1. Edge bimagic total labeling of $K_{1,6} \hat{\mathbf{o}} P_{5}$. 
all $u v \in E$. Now we have to prove that the remaining edges joining $w$ and $x_{i}: 1 \leq i \leq n$ have the common count $k_{2}$.

For any edge $w x_{i}$,

$$
\begin{aligned}
& g(w)+g\left(w x_{i}\right)+g\left(x_{i}\right) \\
& =p+q+p+q+i+p+q+2 n-(i-1) \\
& =3 p+3 q+2 n+1=k_{2} .
\end{aligned}
$$

Thus we have $G^{\prime}=G o ̂ K_{1, n}$ has two common count $k_{1}$ and $k_{2}$. Hence $G o ̂ K_{1, n}$ has edge bimagic total labeling.

Theorem 2.6 If $G$ has super edge edge-magic total labeling then, Gô $F_{1, n}$ admits edge bimagic total labeling.

Proof: Let $G(p, q)$ be super edge edge-magic graph with the bijective function $f: V \cup E \rightarrow\{1,2, \cdots, p+q\}$ such that $f(u)+f(u v)+f(v)=k_{1}$. Let $w \in V$ be the vertex whose label $f(w)=p+q$ is the maximum value. Consider the Fan $F_{1, n}$ with vertex set $\left\{x_{0}, x_{i}: 1 \leq i \leq n\right\}$ and edge set $\left\{x_{0} x_{i}: 1 \leq i \leq n\right\} \cup\left\{x_{i} x_{i+1}: 1 \leq i \leq n-1\right\}$. We superimpose the vertex $x_{0}$ of the Fan $F_{1, n}$ graph on the vertex $w \in V$ of $G$. Now we define the new graph called $G^{\prime}=G \hat{o} F_{1, n}$ with vertex set $V^{\prime}=V \cup\left\{x_{i}: 1 \leq i \leq n\right\}$ and $E^{\prime}=E \cup\left\{w x_{i}: 1 \leq i \leq n\right\} \cup\left\{x_{i} x_{i+1}: 1 \leq i \leq n-1\right\}$. Consider the bijective function $g: V^{\prime} \cup E^{\prime} \rightarrow\{1,2, \cdots, p+q$, $p+q+1, \cdots, p+q+n, \cdots, p+q+2 n-1, \cdots, p+q+3 n-1\}$ defined by $g(v)=f(v)$ for all $v \in V$ and $g(u v)=$ $f(u v)$ for all $u v \in E$.

From our construction of new graph $G$,

$$
f(w)=g\left(x_{0}\right)=g(w)=p+q .
$$

Now

$$
g\left(x_{i}\right)=p+q-1+\frac{1-5(-1)^{i}+6 i}{4}, \text { for } 1 \leq i \leq n .
$$

and $g\left(x_{i} x_{i+1}\right)=p+q+3 n-3 i$, for $1 \leq i \leq n-1$;

$$
g\left(w x_{i}\right)=p+q-1+\frac{12 n+7+5(-1)^{i}-6 i}{4} \text {, for } 1 \leq i \leq n .
$$

Since the graph $G$ is super edge edge-magic with common count $k_{1}$, implies that $g(u)+g(u v)+g(v)=k_{1}$ for all $u v \in E$. Now we have to prove that the remaining edges in the set $\left\{w x_{i}: 1 \leq i \leq n\right\} \cup\left\{x_{i} x_{i+1}: 1 \leq i \leq n-1\right\}$ have the common count $k_{2}$.

For any edge $w x_{i}$,

$$
\begin{aligned}
& g(w)+g\left(w x_{i}\right)+g\left(x_{i}\right)=p+q+p+q-1 \\
& +\frac{12 n+7+5(-1)^{i}-6 i}{4}+p+q-1+\frac{1-5(-1)^{i}+6 i}{4} \\
& =3(p+q+n)=k_{2} .
\end{aligned}
$$

And for the edge $x_{i} x_{i+1}$,

$$
\begin{aligned}
& g\left(x_{i}\right)+g\left(x_{i} x_{i+1}\right)+g\left(x_{i+1}\right)=p+q-1+\frac{1-5(-1)^{i}+6 i}{4} \\
& +p+q+3 n-3 i+p+q-1+\frac{1-5(-1)^{i+1}+6(i+1)}{4} \\
& =3(p+q+n)=k_{2} .
\end{aligned}
$$

Thus we have $G^{\prime}=G \hat{o} F_{1, n}$ has two common count $k_{1}$ and $k_{2}$. Hence $G o ̂ F_{1, n}$ has edge bimagic total labeling.

Example 2.7 illustrates the labeling technique used in the above theorem 2.6.

Example 2.7 Let $k_{1}$ be the constant edge count of an arbitrary graph $G(p, q)$ which is super edge edge-magic with maximum label $p+q=15$ for one of its vertex. The bimagic labeling for $G o ̂ F_{1,5}$ with $k_{2}=3(p+q+n)=60$ can be verified from the given Figure 2.

Theorem 2.8 If $G_{1}$ has super edge edge-magic labeling and $G_{2}$ has super vertex edge-magic labeling then, $G_{1} \hat{o} G_{2}$ admits edge bimagic total labeling.

Proof: Let $G_{1}$ be the super edge edge-magic then there exist the bijective function $f: V_{1} \cup E_{1} \rightarrow\left\{1,2, \cdots, p_{1}+q_{1}\right\}$ such that $f(u)+f(u v)+f(v)=k_{1}$ for all $u, v \in V_{1}$, and Let $G_{2}$ be the super vertex edge-magic then there exist the bijective function $g: V_{2} \cup E_{2} \rightarrow\left\{1,2, \cdots, p_{2}+q_{2}\right\}$ such that $g(u)+g(u v)+g(v)=k_{2}$, for all $u, v \in V_{2}$. Let $w \in V_{1}$ be the vertex whose label is the maximum value $p_{1}+q_{1}$ and $x_{1} \in V_{2}$ be the vertex with label 1 . We superimpose the vertex $x_{1}$ of $G_{2}$ graph on the vertex $w \in V_{1}$. Now we define the new graph called $G^{\prime}=G_{1} \hat{o} G_{2}$ with vertex set $V^{\prime}=V_{1} \cup\left\{V_{2}-x_{1}\right\}$ and $E^{\prime}=E_{1} \cup E_{2}$. Consider the bijective function $h: V^{\prime} \cup E^{\prime} \rightarrow\left\{1,2, \cdots, p_{1}+q_{1}-1\right.$, $\left.p_{1}+q_{1}, p_{1}+q_{1}+1, \cdots, p_{1}+q_{1}+p_{2}+q_{2}\right\}$ defined by

$$
\begin{aligned}
& h(u)=f(u) \text { for all } u \in V^{\prime}\left(G_{1}\right)-w ; \\
& h(u v)=f(u v) \text { for all } u v \in E^{\prime}\left(G_{1}\right) ;
\end{aligned}
$$

$$
h(w)=h\left(x_{1}\right)=p_{1}+q_{1} ;
$$

$h(v)=p_{1}+q_{1}-1+g(v)$ for all $v \in V^{\prime}\left(G_{2}\right)-x_{1}$;

$h(u v)=p_{1}+q_{1}-1+g(u v)$ for all $u v \in E^{\prime}\left(G_{2}\right)$.

For the edges in $G_{1}$, we have

$h(u)+h(u v)+h(v)=f(u)+f(u v)+f(v)=k_{1}$.

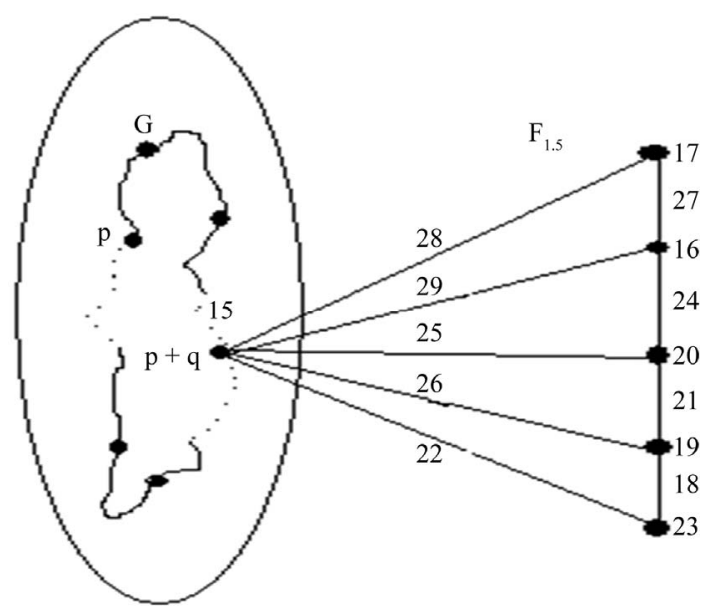

Figure 2. Construction of bimagic for $G \hat{o} F_{1,5}$. 
Since magic labeling is preserved in a graph if all the vertices and edges are increased by any constants, for the edges in $G_{2}$, we have

$$
\begin{aligned}
& h(u)+h(u v)+h(v) \\
= & p_{1}+q_{1}-1+g(u)+p_{1}+q_{1}-1 \\
& +g(u v)+p_{1}+q_{1}-1+g(v) \\
= & 3\left(p_{1}+q_{1}-1\right)+k_{2}=k_{3} .
\end{aligned}
$$

So $G^{\prime}=G_{1} \hat{0} G_{2}$ has two common count $k_{1}$ and $k_{3}$. Hence $G_{1} \hat{o} G_{2}$ admits edge bimagic total labeling.

Theorem 2.9 If $G$ has super edge edge-magic total labeling then, $G+K_{1}$ admits edge bimagic total labeling.

Proof: Let $G(p, q)$ be super edge edge-magic. Then there exist a bijective function function $f: V \cup E \rightarrow\{1,2, \cdots$, $p+q\}$ such that $f(u)+f(u v)+f(v)=k_{1}$. Now we define the new graph called $G^{\prime}=G+K_{1}$ with vertex set $V^{\prime}=V \cup\{x\}$ and $E^{\prime}=E \cup\left\{x v_{i}: 1 \leq i \leq p\right\}$. Consider the bijective function

$g: V^{\prime} \cup E^{\prime} \rightarrow\{1,2, \cdots, p+q, p+q+1, \cdots, 2 p+q+1\}$

defined as follows,

Since there are $p$ vertices in the graph $G$,

$$
\begin{gathered}
g\left(v_{i}\right)=p+q-i+1 \text { for } 1 \leq i \leq p \text { and } \\
g(u v)=f(u v) \text { for all } u v \in E .
\end{gathered}
$$

$g(x)=2 p+q+1$ and $g\left(x v_{i}\right)=p+q+i$; for $1 \leq i \leq p$.

Since the graph $G$ is super edge edge-magic with common count $k_{1}$, implies that $g(u)+g(u v)+g(v)=k_{1}$. Now we have to prove that the remaining $p$ edges joining $V$ and $x$ have the common count $k_{2}$.

For any edge $x v_{i}$,

$$
\begin{aligned}
& g(x)+g\left(x v_{i}\right)+g\left(v_{i}\right) \\
& =2 p+q+1+p+q+i+p+q-i+1 \\
& =4 p+3 q+2=k_{2} .
\end{aligned}
$$

Thus we have $G^{\prime}=G+K_{1}$ has two common count $k_{1}$ and $k_{2}$. Hence $G+K_{1}$ has edge bimagic total labeling.

\section{Concluding Remarks}

Theorem 2.8 shows that $G_{1} \hat{0} G_{2}$ admit edge bimagic total labeling if $G_{1}$ has super edge edge-magic labeling and $G_{2}$ has super vertex edge-magic labeling. Further investigation can be done to obtain the conditions at which $G_{1} \hat{o} G_{2}$ admits edge bimagic total labeling for any two arbitrary total magic graphs.

\section{Acknowledgements}

The referee is gratefully acknowledged for their suggestions that improved the manuscript.

\section{References}

[1] J. A. Gallian, “A Dynamic Survey of Graph Labeling,” Electronic Journal of Combinatorics, Vol. 17, No. 1, 2010, pp. 1-246.

[2] N. Hartsfield and G. Ringel, "Pearls in Graph Theory," Academic Press, Cambridge, 1990.

[3] A. Kotzig and A. Rosa, "Magic Valuations of Finite Graphs,” Canadian Mathematical Bulletin, Vol. 13, 1970, pp. 451-461. doi:10.4153/CMB-1970-084-1

[4] W. D. Wallis, "Magic Graphs,” Birkhauser, Basel, 2001. doi:10.1007/978-1-4612-0123-6

[5] J. B. Babujee, "Bimagic Labeling in Path Graphs," The Mathematics Education, Vol. 38, No. 1, 2004, pp. 12-16.

[6] J. B. Babujee, “On Edge Bimagic Labeling,” Journal of Combinatorics Information \& System Sciences, Vol. 28, No. 1-4, 2004, pp. 239-244.

[7] J. B. Babujee and R. Jagadesh, "Super Edge Bimagic Labeling for Trees” International Journal of Analyzing methods of Components and Combinatorial Biology in Mathematics, Vol. 1 No. 2, 2008, pp. 107-116.

[8] J. B. Babujee and R. Jagadesh, "Super Edge Bimagiclabeling for Graph with Cycles," Pacific-Asian Journal of Mathematics, Vol. 2, No. 1-2, 2008, pp. 113-122.

[9] J. B. Babujee and R. Jagadesh, "Super Edge Bimagic Labeling for Disconnected Graphs," International Journal of Applied Mathematics \& Engineering Sciences, Vol. 2, No. 2, 2008, pp. 171-175.

[10] J. B. Babujee and R. Jagadesh, "Super Edge Bimagic Labeling for Some Class of Connected Graphs Derived from Fundamental Graphs,” International Journal of Combinatorial Graph Theory and Applications, Vol. 1, No. 2, 2008, pp. 85-92. 